\title{
Breadth or conservation score (CS): which is more important for HIV-1 T cell based vaccine immunogen design?
}

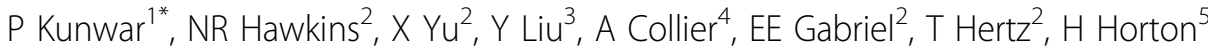 \\ From AIDS Vaccine 2012 \\ Boston, MA, USA. 9-12 September 2012
}

\section{Background}

One of the greatest challenges to develop an efficacious HIV vaccine is the enormous diversity of HIV-1. To tackle this problem, $\mathrm{T}$ cells based vaccine approaches have come up with two main camps: the mosaic immunogen camp: increasing the breadth of vaccine-induced responses, and the conserved immunogen camp: targeting vaccineinduced T-cell responses only to highly conserved viral regions. While both approaches are theoretically sound, there is no current data suggesting that either approach will be successful in inducing $\mathrm{T}$ cells with superior antiviral efficacy. Here we analyzed $\mathrm{T}$ cell responses elicited during early HIV-1 infection, to address the question whether CS of targeted epitopes and breadth of $\mathrm{T}$ cell responses play an important role in viral control.

\section{Methods}

Using IFN- $\gamma$ ELISpot, we comprehensively mapped $\mathrm{T}$ cell epitope specificities recognized by 24 ART-naïve individuals during early infection. We identified CS of targeted epitopes, where the CS is defined as the proportion of random HIV-1 group $M$ amino acid sequences in the LANL database that include the epitope. We used a prediction model to impute the viral load (VL) set-point using the first available VL as a predictor for subjects lacking VL set-point. We further evaluated the association between the CS of the targeted epitopes and breadth of $\mathrm{T}$ cell responses to the individuals' VL set-point.

\section{Results}

The breadth of CD8+ T cell responses inversely correlated with VL set-point $(r=-0.46, p=0.025)$. Subjects possessing

'University of Washington/Seattle Biomedical Research Institute, Seattle, WA,

USA

Full list of author information is available at the end of the article
CD8+ T cells recognizing at least one conserved epitope had a lower VL set-point compared to those recognizing only variable epitopes $(\mathrm{p}=0.093)$.

\section{Conclusion}

Breadth and CS of HIV-specific CD8+ T cells elicited during early infection are both important for controlling viral replication in vivo. Rationale design of immunization approaches should aim at eliciting a greater breadth of CD8+ T cell to conserved epitopes.

This project is funded by NIH grant\#R01AI090783.

\section{Author details}

'University of Washington/Seattle Biomedical Research Institute, Seattle, WA, USA. ${ }^{2}$ Statistical Center for HIV/AIDS Research \& Prevention (SCHARP), Seattle, WA, USA. ${ }^{3}$ Department of Microbiology, University of Washington, Seattle, WA, USA. ${ }^{4}$ Department of Medicine, University of Washington, Seattle, WA, USA. ${ }^{5}$ Seattle Biomedical Research Institute, Seattle, WA, USA.

Published: 13 September 2012

doi:10.1186/1742-4690-9-S2-P260

Cite this article as: Kunwar et al: Breadth or conservation score (CS): which is more important for HIV-1 T cell based vaccine immunogen design? Retrovirology 2012 9(Suppl 2):P260. 